\title{
From Beverages to Biofuels: The Journeys of Ethanol-Producing Microorganisms
}

\author{
Nicholas Macedo and Christopher J. Brigham
}

\author{
Department of Bioengineering University of Massachusetts, Dartmouth, 285 Old Westport Road, North \\ Dartmouth, MA 02747, USA
}

\begin{abstract}
Microbial fermentation for bio-based products is quickly becoming an integral component of the world infrastructure, as the processes encompassing the synthesis of these natural products becomes more efficient and cost effective to compete with existing commodities. Bioethanol is currently one of the most desired fermentation products, as this constituent can be applied to multiple uses in not only contributing to the more traditional routes of beer brewing and winemaking, but also in the foundation for green fuel sources. By optimizing yields, the innovative processes could be applied towards engineering more rapid and productive biomanufacturing. In order to achieve these goals, we as researchers must understand the underlying principles and intricate networks that play a role within the microenvironment and also on the cellular level in key fermentative microbes such as Saccharomyces cerevisiae and Zymomonas mobilis. In-depth pathway analysis could lead to the development of more favorable metabolic outcomes. This review focuses on the key metabolic networks and cellular frameworks in these model organisms, and how biosynthesis of ethanol yields can be optimized throughout the fermentation process.
\end{abstract}

Keywords: Bioethanol, Biofuel, Saccharomyces cerevisiae, Zymomonas mobilis.

\section{INTRODUCTION}

Several different species of the yeast Saccharomyces have been utilized in fermentation processes for many years, and are amenable to genetic manipulation for the construction of strains that have the ability to participate in optimized, higher yield alcohol biofuel manufacturing processes. The brewer's and baker's yeast, Saccharomyces cerevisiae, is the most common and well-studied yeast with applications in the food and beverage industry, molecular genetic research, and biotechnology. Strains of this organism can be grown in either liquid medium or solid agar growth matrices, and typically propagate most efficiently in the presence of dextrose-based nutrient sources in minimal medium containing supplemented essential amino acids, which allows for selective activation of desired promoters for gene expression. Also, increased growth rate has been observed with supplementation of adenine to conventional growth medium [1]. Investigations involving the metabolic reprogramming of this yeast have proven to be successful. S. cerevisiae have the ability to tolerate products of fermentation (i.e., ethanol) to relatively high levels, especially compared to most bacteria. Ethanol, the major product obtained from this metabolic process has been well-studied and shown to be involved in the metabolic maintenance of many Saccharomyces species and strains, as it can be utilized as a feedstock

*Address correspondence to this author at the Department of Bioengineering University of Massachusetts, Dartmouth, 285 Old Westport Road, North Dartmouth, MA 02747, USA; Tel: 1-508-999-9149; Fax: 1-508-999-9139; E-mail: cbrigham@umassd.edu under nutrient deficiency conditions [2]. The importance of studying advances in microbial alcohol productivity stems from the potential that these organisms have in contributing to the area of biologically-produced fuels (Figure 1), while considering improvements in biofuel production concomitant with building a better food industry.

Zymomonas mobilis is a Gram negative, facultatively anaerobic bacterium that has been used for ethanol production in various beverages, although syntheses of compounds that negatively affect flavor have limited its utility in this application. Because $Z$. mobilis grows and ferments rapidly [3], it has also been studied in optimizing ethanol productivity while demonstrating affectivity in producing bioethanol from various carbon sources, mainly hexoses and pentoses like glucose and xylose, via specific fermentative pathways, namely the Entner-Doudoroff pathway [4-5]. Z. mobilis is an interesting organism because it does not utilize oxidative phosphorylation mechanisms [6]. In ethanol fermentation processes, Z. mobilis consumes pentose and hexose sugars for ethanol biosynthesis most efficiently under anaerobic conditions. By products from aerobic growth, such as acetate and lactate accumulating in the growth medium have been observed to decrease ethanol production significantly compared to anaerobic conditions [7]. However, this bacterium has been reported to be tolerant to ethanol byproducts from fermentation, which is a unique attribute among bacteria. Z. mobilis has been shown to have a high sugar uptake rate, high ethanol production rate, and tolerance to high concentrations of ethanol 


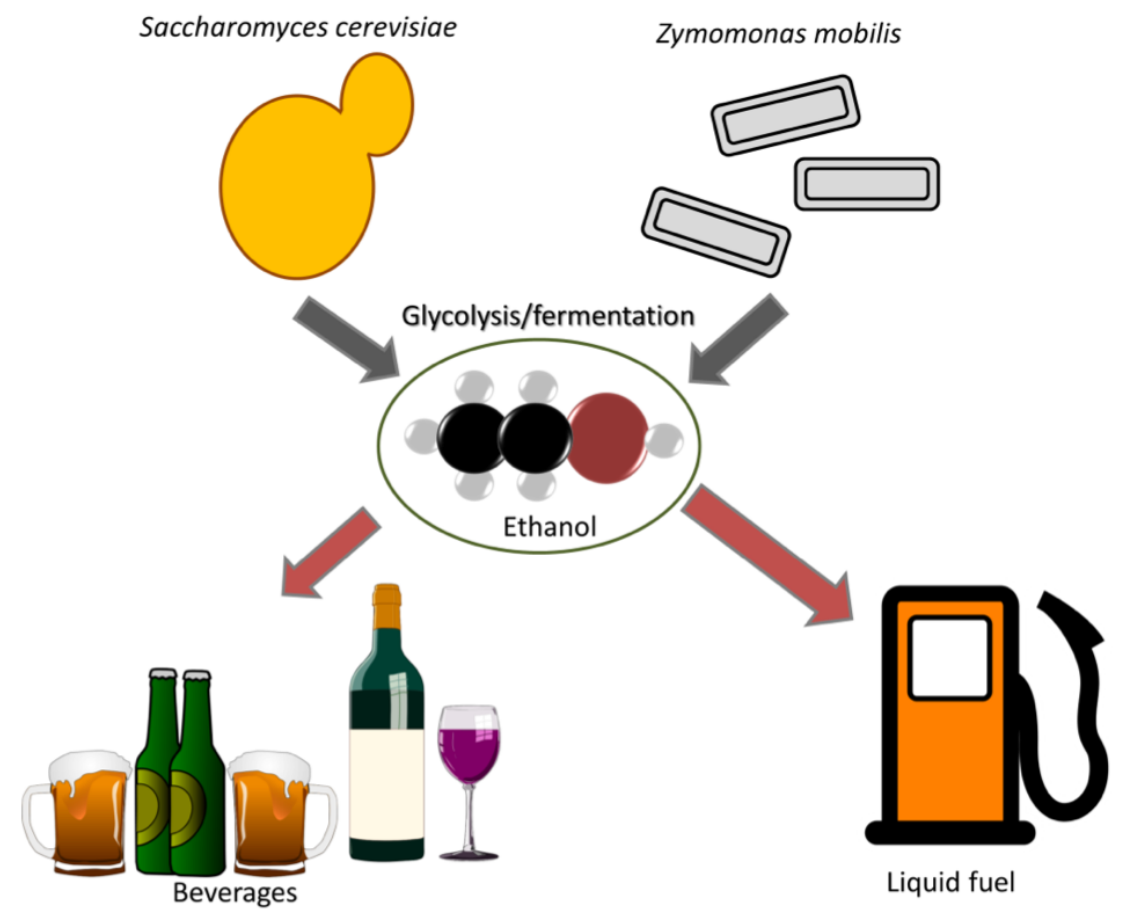

Figure 1: General schematic of ethanol production by the yeast Saccharomyces cerevisiae and Zymomonas mobilis. S. cerevisiae ethanol production has been utilized for centuries in order to produce beverages like beer or wine. Z. mobilis has been used for alcoholic beverage production (e.g., palm wine), but is generally regarded as a contaminant in traditional wine and beer manufacture. Researchers and industry have been utilizing the ethanol biosynthetic capabilities of both organisms to produce biofuels from carbohydrate feedstocks. Clip art obtained from openclipart.org.

$(>16 \%, v / v)[8]$. Regarding the latter attribute, a wholegenome transcriptome analysis of $Z$. mobilis has indicated that a complex mechanism of tolerance to ethanol is employed, including expression of genes involved in transport of solutes, hopanoid and terpenoid biosynthesis, and DNA recombination/repair, among others [9].

\section{Applications in Brewing and Winemaking}

In winemaking and brewing, the species of yeast most commonly selected is $S$. cerevisiae, being the most extensively studied species of yeast by fermentation scientists, while also being the organism of choice for more rapid bioethanol production. In beer maturation from initial wort, barley proteins contribute to the overall proteome of the final product caused by yeast-mediated synthesis of desirable peptides such as lipid transfer protein 1 (LTP1), which serves as an antioxidant in brews and has been experimentally confirmed to be produced as a byproduct of $S$. cerevisiae fermentation in both laboratory and brewery processes [10]. This species of yeast is a known model for genetic manipulation of the cellular machinery in the interest of optimizing production of ethanol from the fermentation process and engineering a better brew [11].
The traditional wine making process that takes place at the vineyards of Sicily has been studied for a diverse population of yeasts including various strains of $S$. cerevisiae, and genomic analysis has allowed elucidation of the cause of variations in chemical makeup of the wine and also in overall ethanol yield [12]. Over the course of the fermentation process, this yeast has been demonstrated to be quite robust in terms of resistance to byproducts that would normally be cytotoxic to other microbes (Figure 2). However, levels of ethanol above $50 \%(\mathrm{v} / \mathrm{v})$ produced in the process affect membrane fluidity and can result in solubilizing of membrane lipids, thus diminishing membrane integrity. In response to this, $S$. cerevisiae has been known to express several heat shock proteins (HSPs) in order to enable tolerance to the ethanol produced [13]. Transcripts of these proteins have been known to be overexpressed during these conditions, and follow the establishment of intracellular $\mathrm{pH}$ stability during oxidative stress to the cellular membrane. Studies involving the identification of glucose transport complexes in particular have clearly indicated that these detoxification mechanisms in ethanol-resistant strains are vital for the survival of the yeast, and mutants that did not express these extracellular transport proteins were observed to be sensitive to ethanol exposure. 


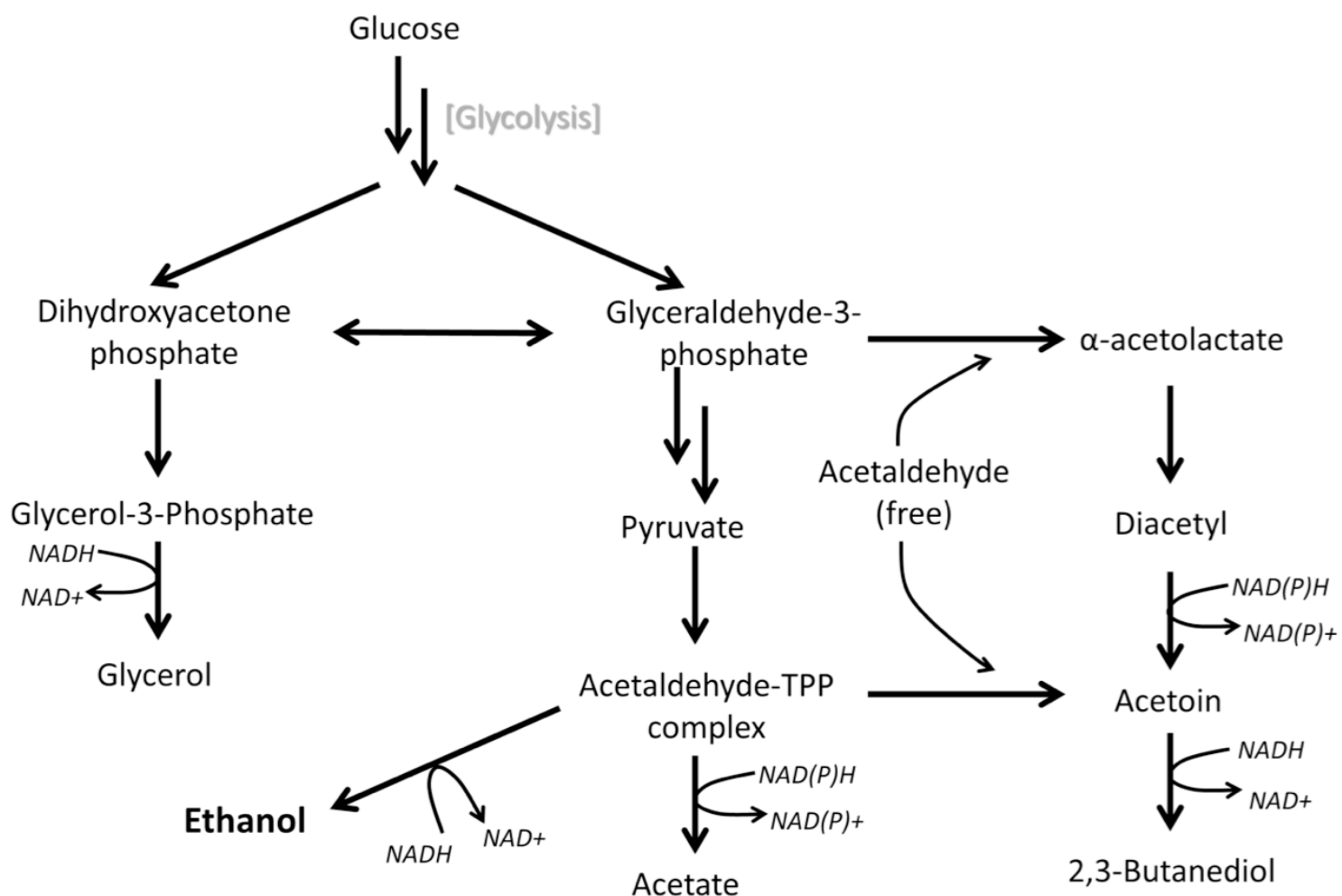

Figure 2: Ethanol biosynthesis pathway in Saccharomyces cerevisiae. The by-products, glycerol, 2,3-butanediol, acetoin, and acetate are also produced in quantities smaller than that of ethanol. These by-products often lend flavor characteristics to alcoholic beverages. In biofuels production, researchers often attempt to minimize carbon flux to glycerol and 2,3-butanediol in order to maximize ethanol productivity.

Zymomonas mobilis strains have been involved in the fermentation processes encompassing brewing and beer making. However, this species recently has been known as a contaminant to the fermentation mix. The bacteria have been known to form surface colonies in the brew, and gradually matriculate deeper where they produce almost $1.9 \mathrm{~mol}$ of ethanol for every $1 \mathrm{~mol}$ of glucose present [14]. Current research has shown that Z. mobilis favors a pathway in metabolism that reflects the overall yield of the microbial products, and utilizes a pathway that dominates the determining factors in ethanol production by NADH-dependent activation of alcohol dehydrogenase (Figure 3), suggesting that ATP hydrolysis does not play a prominent role in the production of ethanol in this species of bacteria [15].

\section{THE ECONOMICS OF ETHANOL}

In recent years, ethanol production levels have reached their highest recorded $(940,000$ barrels/day in summer of 2014) in the United States [16]. Even with such high production levels, the price of ethanol recently was at its highest since 2006 (from US $\$ 3.80$ 3.99 , as of early 2014), due to increased demand for biofuels and the resulting logistics to deliver ethanol to the consumer [17]. While ethanol biofuels have had their share of growing pains in the United States, 44 years of infrastructure development have resulted in Brazil taking a leading edge position in ethanol biofuel production and consumption [18]. Currently, in Brazil all light vehicles (passenger cars, etc.) run on a flexible fuel blend of gasoline and ethanol [19]. Ethanol consumption as a fuel is predicted to continue rising, in the face of concerns about crude oil (e.g., environmental impact and rising prices). Moving forward, if ethanol production is to compete economically with petroleum fuels, then inexpensive feedstocks will have to be utilized. Currently, the most cost-effective feedstocks are biomass (waste) and South American sugarcane [20]. Waste biomasses (discussed below) would be the ideal biofuel feedstock, avoiding any "food v. fuel" controversy. The edible carbon feedstocks can (and will) be used for production of alcoholic beverages, as they have been for centuries before.

\section{BIOFUEL PRODUCTION}

Apart from its role in fermentation of beverages, $S$. cerevisiae yeast is well known for its genetic tractability, allowing for manipulation of metabolic pathways involved in the production of biofuels using 


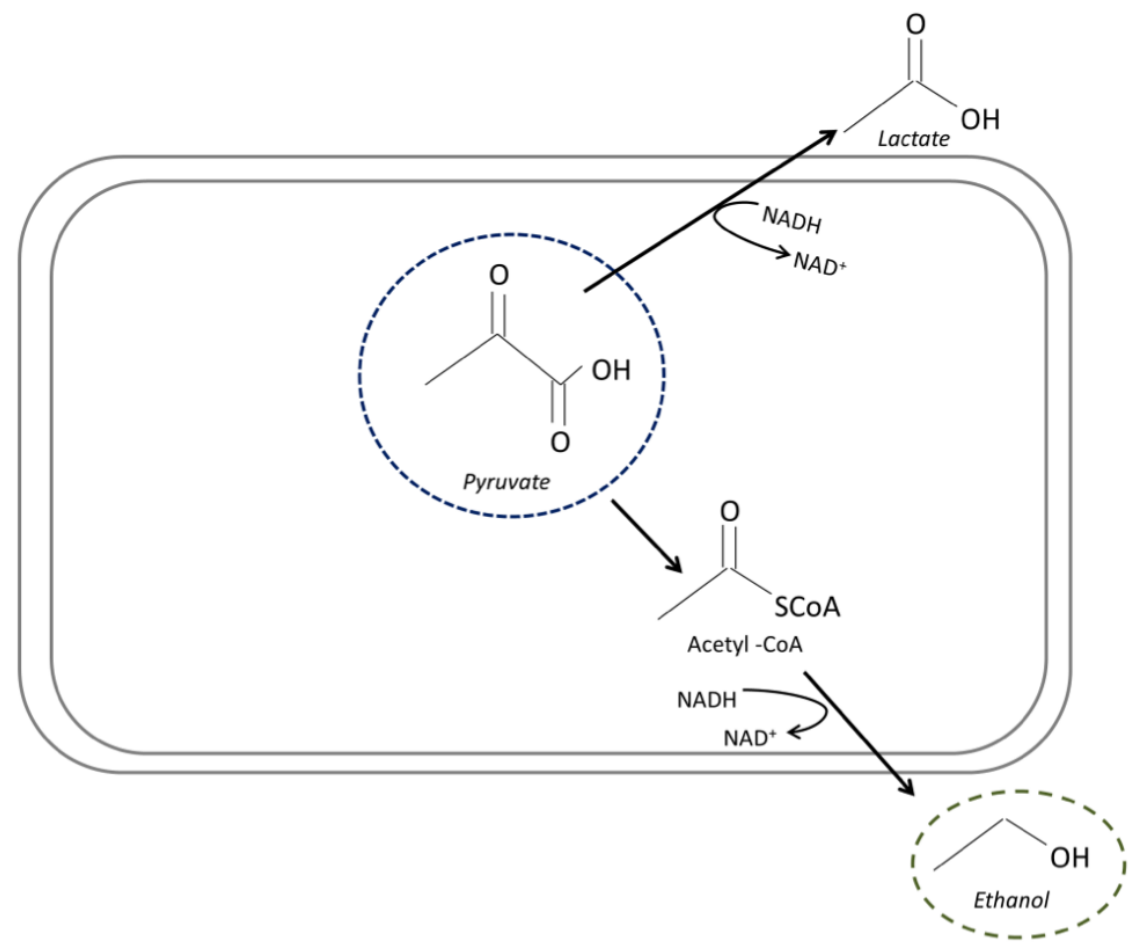

Figure 3: After initial glycolysis, at the branched metabolic path from pyruvate, Zymomonas. mobilis favors an NADH- dependent ethanol production cycle, as discussed in [15]. Also, the biosynthesis of lactate, a by-product in Z. mobilis ethanol fermentation, is NADH-dependent.

glucose and other feedstocks. For example, it can be easily altered to maximize ethanol yield and productivity at the end of fermentation processes using xylose in conjunction with cellobiose as the carbon sources [21]. A challenge that is faced by industry for efficient biofuel production is the elimination of a number of compounds found in feedstock pretreatments that inhibit metabolism by toxic means. It is important and advantageous to utilize an organism that can tolerate such conditions, and industrial strains of yeasts are often developed to bypass these issues in production. Furan derivatives are regularly found in pretreated lignocellulosic feedstock. Other aromatic compounds, such as the phenolic aldehyde known as vanillin and a 4-hydroxybenzaldehyde isomer, both also commonly found in vanilla extract, and various types of wood constituent compounds have been demonstrated to decrease overall ethanol yield in batch processes due to the growth inhibition by the presence of reactive oxygen species (ROS) in various yeast species [22]. Genes involved in detoxification processes in S. cerevisiae play the most significant role in adapting the microorganism for using varieties of different, naturally abundant feedstock to optimize ethanol yield. The process involves a cluster of genes for deactivating the toxic metabolite 5hydroxymethylfurfural (HMF) from the breakdown of lignocellulose-based feedstock. As a result, the presence of HMF in growth and ethanol production media has been shown ultimately to increase the lag phase of growth during the fermentation process [23]. The genetic damage repair proteins encoded by MAG1 and DD/1 genes are HMF-inducible as they are sequentially activated in response to DNA damage and act on SON1 promoter sequences, which induce mRNA binding complexes to signal for enhanced regulatory control in gene repair by activating splicing mechanisms. In terms of transcript degradation, arginase enzymes encoded by 8 different variants of the $A R G$ gene in arginine biosynthesis have been experimentally proven to be downregulated by HMF byproducts, thus inhibiting growth in $S$. cerevisiae. To overcome these metabolic challenges, research has focused on introducing factors for enhanced detoxification of these byproducts of lignocellulose catabolism, and research groups have shown that the introduction of a variety of transcription factors, such as HSF1 and members from the bacterial PDR (pleiotropic drug resistance) family into the yeast genome can significantly alter the aforementioned cellular responses in the lag phase of growth [23], thus increasing cellular resistance to these damaging byproducts. In a recent study, the overexpression of the YAP1 transcription factor and MCR1, encoding the 
mitochondrial $\mathrm{NADH}$-cytochrome b5 reductase, in $\mathrm{S}$. cerevisiae strains grown on pretreated lignocellulose resulted in greater detoxification of HMF and furfural [24].

Focus on production of bioethanol has generally relied on the optimized metabolic behavior of $S$. cerevisiae being able to metabolize $D$-xylose, which is an abundant sugar in lignocellulose-based feedstock. This metabolic pathway has been augmented by the introduction of xylose isomerase genes into the genome, resulting in active processing of the sugar. The cloning of the isomerase genes from bacterial genomes has been reported to be successful with ethanol yields from engineered $S$. cerevisiae strains reaching about $13.6 \mathrm{~g} / \mathrm{L}$ in $91 \mathrm{~h}$ [25] despite the recent failed efforts of other researchers due to translation of inactive enzymes partly resulting from incompatibility between the species of microbes.

Antifungal agents derived from essential oils, such as eugenol and carvol, have been studied extensively due to their ability to inhibit formation of spores by exploiting the pathways in the fungi, causing dysregulation of intracellular ionic homeostasis. This mechanism has been studied and correlated to the increased signaling of $\mathrm{Ca}^{2+}$ across the plasma membrane in model organisms including yeast strains, and contributes to the protection against essential oil toxicity through pathways dependent on the plasma membrane $\mathrm{Ca}^{2+}$ channel, $\mathrm{CCH} 1 \mathrm{p}$ [26]. This increase in cellular signaling could be exploited to ultimately produce a wider variety of microbial products, as a mechanism introduced in the process could capitalize on this activated state of mitochondrial activity to metabolize nutrients more rapidly because of the inductive nature of nutrient starvation in yeasts like $S$. cerevisiae [27]. One group was able to use eugenol as a feedstock for the production of coniferyl alcohol by introducing a gene native to Penicillium simplicissimum called the vaoA gene, which encodes for the enzyme flavoenzyme vanillyl-alcohol oxidase. The resulting strain produced alcohol products up to $16.9 \mathrm{~g} / \mathrm{L}$ after a few days of culture [28]. This suggests the ability to develop these essential oils as constituents in growth media to signal for greater cellular activity of resistant strains of yeast for the use in alcohol production.

Isobutanol, as a biofuel, can be incorporated into gasoline and diesel fuels up to $85 \%$ due to its longer carbon chain length and favorable combustion properties, and is commercialized by Butalco (www.butalco.com), Butamax (www.butamax.com) and
Gevo (www.gevo.com) and the efforts invested in yeast mitochondrial function and activity have provided the critical role in optimized isobutanol production [29]. S. cerevisiae has also been identified as a viable biocatalyst for the production of isobutanol biofuel. One study has shown that the overexpression of the genes controlling mitochondrial energy production via the activation of the isoleucine, leucine and valine (ILV) gene family involved in branched chain amino acid biosynthesis have a significant role in altering the potential for this organism to produce isobutanol. Deletions of these genes have been shown experimentally to alter metabolism involving the synthesis of a metabolic intermediate of the amino acid valine known as ketoisovalerate, which is a critical factor inducing metabolic uptake of nutrients in this process [29]. Also, decarboxylation mechanisms involved in these biosynthesis pathways largely contribute to a similar mechanism participating in keto acid decarboxylation in the synthesis and exporting of isobutanol, and there are specific genes involved in these processes that contribute to its production. Metabolic pathways have been engineered around the heterologously-expressed kivd gene (from Lactococcus lactis), which encodes for one such decarboxylation enzyme used in biofuel metabolism in conversions from pyruvate and other metabolic intermediates to alcohol byproducts. The divergence to higher alcohols in the pathway occurs in the pyruvate metabolic path to ethanol, where intermediates are oxidized to a 2-keto species before participating in catalysis to form, for example, isobutanol [30] (Figure 4).

Z. mobilis has also been examined for production of biofuels, and has been improved by various genomic alterations in metabolic pathways to ultimately produce more ethanol with yields greater than those of some yeast strains [31], but more recent research has shown that this bacterium can be utilized in for production of bioethanol using xylose as the primary nutrient in a carbon feedstock. This has relevance in considering lignocellulose as a feedstock for biofuels production using Z. mobilis as the biocatalyst. In experiments involving serial exposure to acetate and glucose alone over a gradual period, one group demonstrated the ability of $Z$. mobilis to increase its capacity to utilize xylose in a modified strain. As a result, ethanol was produced at a yield of $30 \mathrm{~g} / \mathrm{L}$ over the course of 3 days by inducing a transcriptional regulator known as ZMO0774 [32]. Further profiling of this pathway revealed that genes encoding for glucokinase, phosphoglucono-lactonase, phosphoglycerate kinase 


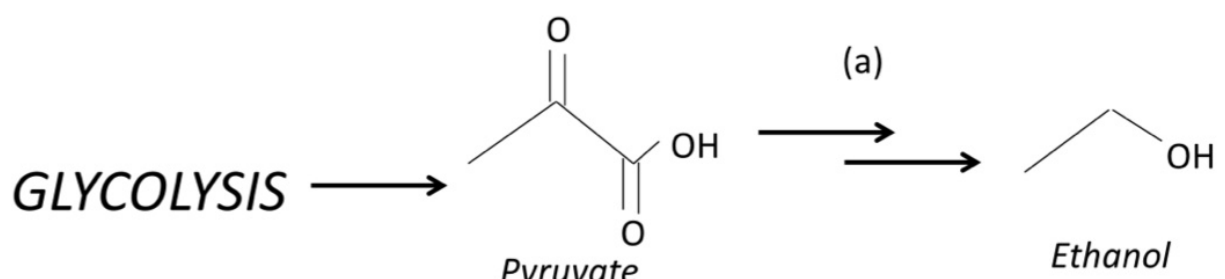

Pyruvate

Ethanol

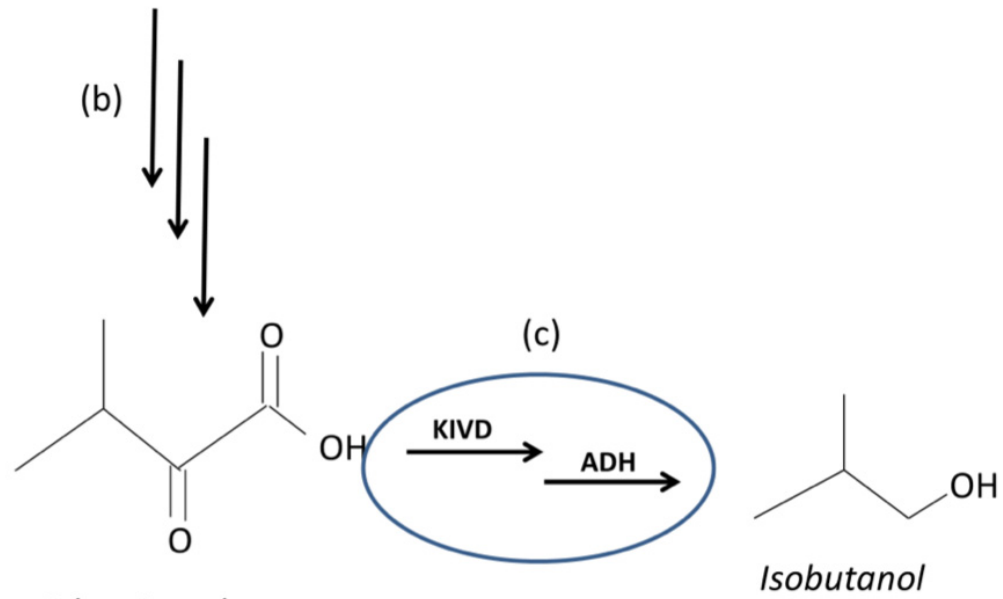

\section{2-ketoisovalerate}

Isobutanol

Figure 4: Branching from the pyruvate node to produce higher alcohols. Wild-type S. cerevisiae and Z. mobilis can produce ethanol from pyruvate (a), using pathways similar to that which is outlined in Figure 2 . The native branched-chain amino acid pathway will produce the intermediate compound 2-ketoisovalerate from pyruvate (b), in order to synthesize the essential building block valine. The addition of the kivd gene, which encodes an enzyme (KIVD) that acts in the decarboxylation of 2-keto intermediates that are in turn converted to higher alcohol byproducts by an alcohol dehydrogenase (ADH) (c).

and enolase, all of which are enzymes participating in the Entner-Doudoroff pathway, were highly transcribed during peaks in productivity, which, in turn, increased the production of both protective proteins that act to maintain cell membrane integrity and hopanoids, which form a unique cell membrane structure that is alcohol resistant [33]. As the activation of native metabolic pathways in bacteria to increase energy production rates do not typically enable direct production of biofuels like ethanol, the ability of cells under stress from cytotoxic byproducts to utilize more freely available nutrients in order to produce protective agents against toxic compounds will provide the resistance needed to maintain normal growth conditions when harmful fermentation byproducts are more steadily produced over the course of the process. These studies indicate that heterologous pathways may be needed for engineering more efficient feedstock utilization in such fermentation conditions, for various species of microorganisms.

\section{PRODUCTION PROCESSES}

With the need for more efficient and sustainable fuel sources, the design of new methods for synthesizing biofuels must be considered, in that large scale production of such fuels requires optimized continuous processing. Governments have pushed industries toward biofuel production to decrease the amount of carbon dioxide emissions from the combustion of conventional fossil fuels. Therefore, a large manufacturing initiative is currently geared toward exploiting microbial biosynthesis capabilities through studies involving a variety of techniques to evaluate the most efficient components necessary to achieve a high yield for downstream processing and refining. Biodiesel is being adopted as a popular fuel product in Europe, and has been produced from animal fats, essential oils, and other highly dense methyl ester compounds resulting from transesterfication chemical synthesis. In Brazil, industries have optimized processes for utilizing sugarcane, which can be produced in large yields, for feedstock in fermentation protocols using engineered strains of yeast and other microorganisms, which ultimately offers a renewable, cleaner alternative for producing such biofuels.

One method by which ethanol biofuel production processes have been optimized is by utilizing the pyruvate decarboxylase $(p d c)$ and alcohol dehydrogenase II (adh II) genes from Z. mobilis 
integrated into Escherichia coli strains to produce engineered organisms capable of metabolizing glucose under anaerobic conditions more effectively using hexose sugars as carbon feedstocks. The further introduction of a fumarate reductase gene (frd) mutation in the engineered $E$. coli strain resulted in yields of up to $95 \%$ on glucose in complex media [34].

Another Gram-negative bacterium, Klebsiella. oxytoca, has been engineered for industrial production of ethanol with yields around $45 \mathrm{~g} / \mathrm{L}$ after 2 days from the initiation of fermentation mix exposure. by integrating an operon termed PET (production of ethanol) into the genome. This operon consists of the aforementioned adh $/ /$ and pdc genes, under the control of the $\mathrm{P}_{\text {lac }}$ promoter, and has since been tested in the metabolizing of paper wastes and cellobiose to ethanol, while also demonstrating hyper- resistance towards the fermentation product, ethanol [35].

\section{BUILDING A BETTER FEEDSTOCK}

In fertilizers, phosphorous has been used as a component in feedstock for use in the production pathways of soil bacteria and overall crop development. Aside from the food industry, phosphorous is also a primary feedstock and essential nutrient for biodiesel production. The conflicting need for this nutrient between these two processes alone can ultimately impact the future of the global food industry. Therefore, it is important to engineer alternative feedstocks separately for biofuel production as the recycling efficiency of phosphorous is too low to consider reuse of the nutrient [36]. Lignocellulose is a readily available feedstock for biofuel production, as it can be obtained from a variety of plant sources because of the polymer's natural abundance in plant cell walls. It is composed of cellulose, hemicellulose and lignin, most of which can be broken down and then utilized by a variety of bacteria as fermentable sugars for cellular processing. However, pentose sugars often cannot be metabolized by fermenting organisms [5, 37]. However, in the establishment of these feedstock mixtures, productivity is often very low due to physical inability for the enzymes to break down large amounts of lignin in initial cellulosic nutrient stocks. This issue has been overcome by incorporating laccasemediator/separator mechanisms in order to rid lignin from the cellular wall components in the cellulose, and prior research has demonstrated that by enzymatic hydrolysis with this mediator, they could obtain yields of xylose upward of $37 \%$ in fermentation conditions using recombinant Myceliophthora thermophila [38], which could then be utilized for yeast fermentation to produce bioethanol. Another interesting feedstock for use in fermentation processes for producing bioethanol is lactose, as it is the abundant constituent in cheese whey and is a byproduct in these dairy-based production methods. Lactose can be used by other microorganisms that contain the metabolic lacZ gene, but the yeasts that are involved in common industrial mass fermentation processes do not conserve the ability to metabolize lactate efficiently. However, galactose is regularly utilized by $S$. cerevisiae using the GAL intracellular protein transport system, which can be altered in function by introduction of the GAL4 transcriptional activator. This, in turn, has resulted in ethanol in yields ranging from 73- 84\% [39]. In combination with this cost-efficient feedstock and genetic engineering of microorganisms, researchers can provide more viable processes for producing maximal biofuel products.

\section{CONCLUSION}

As biotechnologists, we adapt and discover mechanisms of action in an efficient manner to determine a cause that results in the desired effects in a biological role or system. In the ethanol producing organisms S. cerevisiae and Z. mobilis, it is a reality that the metabolic pathways and activities are well known, prevalent in a number of organisms, and easily manipulated in both laboratory and large scale productions of ethanol and other valuable products. The future holds promise for the efficiency in production processes from these microorganisms, and could potentially lead to more renewable and ecofriendly fuel sources for motor vehicles and other traditionally "fossil fuel-powered" machines. The importance in developing these molecular pathways are crucial in providing future research with the ability to study more in depth applications and uses for these organisms and greatly reduce the need for current petrochemical-based fuel sources.

\section{REFERENCES}

[1] Saghbini M, Hoekstra D, Gautsch J. Media Formulations for Various Two-Hybrid Systems. Method Mol Biol 2011; 177: 15-39.

[2] Piškur J, Rozpedowska E, Polakova S, et al. How did Saccharomyces evolve to become a good brewer? Trends Genet 2006; 22(4): 183-6. http://dx.doi.org/10.1016/j.tig.2006.02.002

[3] Jeffries TW. Ethanol fermentation on the move. Nat Biotechnol 2005; 23: 40-1. http://dx.doi.org/10.1038/nbt0105-40

[4] Dunn KL, Rao CV. Expression of a xylose-specific transporter improves ethanol production by metabolically 
engineered Zymomonas mobilis. Appl Microbiol Biotechnol 2014; 98(15): 6897-905

http://dx.doi.org/10.1007/s00253-014-5812-6

[5] Lee KY, Park JM, Kim TY, Yun H, Lee SY. The genomescale metabolic network analysis of Zymomonas mobilis ZM4 explains physiological features and suggests ethanol and succinic acid production strategies. Microb Cell Fact 2010; 9: $1-12$.

http://dx.doi.org/10.1186/1475-2859-9-94

[6] Ingram LO, Conway T, Clark DP, Sewell GW, Preston JF. Genetic engineering of ethanol production in Escherichia coli. Appl Environ Microbiol 1987; 53(10): 2420-5.

[7] Yang S, Tschaplinski TJ, Engle NL, et al. Transcriptomic and metabolomic profiling of Zymomonas mobilis during aerobic and anaerobic fermentations. BMC Genomics 2009; 10(34). doi:10.1186/1471-2164-10-34.

[8] Seo JS, Chong H, Park HS, et al. The genome sequence of the ethanologenic bacterium Zymomonas mobilis. Nat Biotechnol 2005; 23(1): 63-8.

http://dx.doi.org/10.1038/nbt1045

[9] Ming-Xiong $\mathrm{H}, \mathrm{Wu} \mathrm{B}$, Zong-Xia $\mathrm{S}$, et al. Transcriptome profiling of Zymomonas mobilis under ethanol stress. Biotechnol Biofuels 2013; 5(75): 70-6. doi:10.1186/17546834-5-75.

[10] Berner TS, Jacobsen S, Armstrong N. The impact of different ale brewer's yeast strains on the proteome of immature beer. BMC Microbiology 2013; 13: 1-8. doi:10.1186/1471-2180-13215.

[11] Tristezza M, Fantastico L, Vetrano C, Bleve G, Corallo D, Grieco F, Mita G, Grieco F. Molecular and Technological Characterization of Saccharomyces cerevisiae Strains Isolated from Natural Fermentation of Susumaniello Grape Must in Apulia, Southern Italy. Int J Microbiol 2014; 1-11. doi.org/10.1155/2014/897428.

[12] Di Maio S, Polizzotto G, Di Gangi E, Foresta G, Genna G, et al. Biodiversity of Indigenous Saccharomyces Populations from Old Wineries of South-Eastern Sicily (Italy): Preservation and Economic Potential. PLoS One 2012; 7(2): e30428.

http://dx.doi.org/10.1371/journal.pone.0030428

[13] Stanley D, Bandara A, Fraser S, Chambers PJ, Stanley GA. The ethanol stress response and ethanol tolerance of Saccharomyces cerevisiae. J Appl Microbiol 2009; 109: 1324. doi:10.1111/j.1365-2672.2009.04657.x.

[14] Swings J, De Ley J. The Biology of Zymomonas. Bacteriol Rev 1977; 41(1): 1-46.

[15] Kalnenieks U, Galinina N, Toma MM, Marjutina U. Ethanol cycle in an ethanologenic bacterium. FEBS Lett 2002; 522: 6-8. http://dx.doi.org/10.1016/S0014-5793(02)02923-X

[16] Voegele E. Recent ethanol production volumes among highest recorded levels. Ethanol Producer [serial on the internet]. 2014 August 15 [about 2 screens]. Available from: http://www.ethanolproducer.com/articles/11356/recentethanol-production-volumes-among-highest-recorded-levels

[17] Parker M. Ethanol rises to highest price since July 2006. Bloomberg News [serial on the internet]. 2014 March 28 [about 4 screens]. Available from: http://www.bloomberg. com/news/2014-03-28/ethanol-rises-to-highest-price-sincejuly-2006.html

[18] Rothkopf G. A blueprint for green energy in the Americas. Inter-American Development Bank. Washington, D.C.; 2007.

[19] Puerto Rico JA. Programa de Biocombustíveis no Brasil e na Colômbia: uma análise da implantação, resultados e perspectivas. Ph.D. Thesis. Universidade de Sao Paulo, Brazil 2008.

[20] Miranowski J, Rosburg A. Long-term biofuel projections under different oil price scenarios. AgBioForum 2012; 16(1): 79-87.
[21] $\mathrm{Ha}$ SJ, Galazka JM, Kim SR, et al. Engineered Saccharomyces cerevisiae capable of simultaneous cellobiose and xylose fermentation. Proc Nat Acad Sci USA 2011; 108 (2): 504-9.

http://dx.doi.org/10.1073/pnas.1010456108

[22] Hawkins GM, Doran-Peterson J. A strain of Saccharomyces cerevisiae evolved for fermentation of lignocellulosic biomass displays improved growth and fermentative ability in high solids concentrations and in the presence of inhibitory compounds. Biotechnol Biofuels 2011; 4(49): 1-14. doi:10.1186/1754-6834-4-49.

[23] Ma M, Liu ZL. Comparative transcriptome profiling analyses during the lag phase uncover YAP1, PDR1, PDR3, RPN4, and HSF1 as key regulatory genes in genomic adaptation to the lignocellulose derived inhibitor HMF for Saccharomyces cerevisiae. BMC Genom 2010; 11(660): 480-8. doi:10.1186/1471-2164-11-660.

[24] Salinas VW, Signori L, et al. Re-assessment of YAP1 and MCR1 contributions to inhibitor tolerance in robust engineered Saccharomyces cerevisiae fermenting undetoxified lignocellulosic hydrolysate. AMB Express 2014; 4: 56.

\section{http://dx.doi.org/10.1186/s13568-014-0056-5}

[25] Hector RE, Dien BS, Cotta MA, Mertens JA. Growth and fermentation of D-xylose by Saccharomyces cerevisiae expressing a novel $\mathrm{D}$-xylose isomerase originating from the bacterium Prevotella ruminicola TC2-24. Biotechnol Biofuels 2013; 6: 84 .

http://dx.doi.org/10.1186/1754-6834-6-84

[26] Roberts SK, McAinsh M, Widdicks L. Cch1p Mediates Ca2+ Influx to Protect Saccharomyces cerevisiae against Eugenol Toxicity. PLoS One 2012; 7(9): 1-7. doi:10.1371/ journal.pone.0043989.

[27] Zhang Y, Muend S, Rao R. Dysregulation of ion homeostasis by antifungal agents. Front Microbiol 2012; 3(133): 1-6. doi: 10.3389/fmicb.2012.00133

[28] Lambert F, Zucca J, Ness F, Aigle M. Production of ferulic acid and coniferyl alcohol by conversion of eugenol using a recombinant strain of Saccharomyces cerevisiae. Flavor Fragr J 2013; 29: 14-21. http://dx.doi.org/10.1002/ffj.3173

[29] Buijs NA, Siewers V, Nielsen J. Advanced biofuel production by the yeast Saccharomyces cerevisiae. Curr Opin Chem Biol 2012; 17: 480-8. http://dx.doi.org/10.1016/j.cbpa.2013.03.036

[30] Zhang F, Rodriquez S, Keasling JD. Metabolic engineering of microbial pathways for advanced biofuels production. Curr Opin Biotechnol 2011; 22: 775-83. http://dx.doi.org/10.1016/j.copbio.2011.04.024

[31] Loos H, Kramer R, Sahm H, Sprenger GA. Sorbitol Promotes Growth of Zymomonas mobilis in Environments with High Concentrations of Sugar: Evidence for a Physiological Function of Glucose-Fructose Oxidoreductase in Osmoprotection. J Bacteriol 1994; 176(24): 7688-93.

[32] Mohagheghi A, Linger J, Smith $\mathrm{H}$, et al. Improving xylose utilization by recombinant Zymomonas mobilis strain $8 \mathrm{~b}$ through adaptation using 2-deoxyglucose. Biotechnol Biofuels 2014; 7(19): 1-9. doi:10.1186/1754-6834-7-19.

[33] He MX, Wu B, Shui ZX, et al. Transcriptome profiling of Zymomonas mobilis under ethanol stress. Biotechnol Biofuels 2012; 5(75): 1-10. doi:10.1186/1754-6834-5-75.

[34] Peterson JD, Ingram LO. Anaerobic Respiration in Engineered Escherichia coli with an Internal Electron Acceptor to Produce Fuel Ethanol. Ann NY Acad Sci 2008; 1125: 363-72. http://dx.doi.org/10.1196/annals. 1419.020

[35] Dien BS, Cotta MA, Jeffries TW. Bacteria engineered for fuel ethanol production: current status. Appl Microbiol Biotechnol 2003; 63: 258-66.

http://dx.doi.org/10.1007/s00253-003-1444-y 
[36] Hein L, Leemans R. The Impact of first-Generation biofuels on the depletion of the global phosphorus reserve. AMBIO 2012; 41: 341-9. http://dx.doi.org/10.1007/s13280-012-0253-x

[37] Moraïs S, Morag E, Barak $Y$, et al. Deconstruction of lignocellulose into soluble sugars by native and designer cellulosomes. MBio 2012; 3(6): e00508-12. http://dx.doi.org/10.1128/mBio.00508-12
[38] Rico A, Rencoret J, Rio J, et al. Pretreatment with laccase and a phenolic mediator degrades lignin and enhances saccharification of Eucalyptus feedstock. Biotechnol Biofuels 2014; 7(6): 1-14. doi:10.1186/1754-6834-7-6.

[39] Domingues L, Guimarães PMR, Oliveira C. Metabolic engineering of Saccharomyces cerevisiae for lactose/whey fermentation. Bioeng Bugs 2010; 1(3): 64-171. http://dx.doi.org/10.4161/bbug.1.3.10619

Received on 05-08-2014

DOI: http://dx.doi.org/10.6000/1927-3037.2014.03.03.1

(c) 2014 Macedo and Brigham; Licensee Lifescience Global.

This is an open access article licensed under the terms of the Creative Commons Attribution Non-Commercial License (http://creativecommons.org/licenses/by-nc/3.0/) which permits unrestricted, non-commercial use, distribution and reproduction in any medium, provided the work is properly cited. 